\title{
FLUID INJECTIONS IN DEHYDRATED INFANTS *
}

\author{
STAFFORD MCLEAN AND CHARLES A. LANG, M.D. \\ NEW YORK
}

During the summer of 1918 we made an effort to secure some exact clinical data on the effects of introduction of fluids in the case of dehydrated infants. This work was started with the idea of using only the peritoneal route as described by Blackfan. ${ }^{1}$ Preliminary to the introduction of his method into routine use, we made a number of intraperitoneal injections in a series of six rabbits. Sixty c.c. of 6 per cent. dextrose solution was used in one group of three rabbits and 60 c.c. of 4 per cent. sodium bicarbonate solution was used in another group of three rabbits. Careful records were kept of the animals after treatment, as to temperature, activity, appetite, etc. In three rabbits we used a solution of 6 per cent. dextrose combined with 4 per cent. sodium bicarbonate. The six animals received forty injections in all. There was one death. This animal died after the third injection of 60 c.c. of a 4 per cent. bicarbonate solution. The necropsy findings were as follows : 25 c.c. of a chocolate colored fluid was found in the peritoneal cavity which showed microscopically a few grampositive organisms. The culture was sterile. There was no peritonitis and no puncture of the intestines was discovered. The rabbits were caged in a very small room, the weather was very hot with high humidity so that factors other than the abdominal condition may have been the cause of death.

It was our feeling after the experiments with the rabbits that similar injections could be given to infants without causing either injury or discomfort. We prepared to give most of our dehydrated infants fluids by the intraperitoneal route, but in many cases this was not done for reasons having no relation to this study.

A special chart was kept in each ward where the fluids were administered (Fig. 1). In conjunction with the ordinary ward records data relating to the immediate effects of fluids injected were also recorded; these included weight taken before injection and twenty-four hours later and records of pulse, temperature and respiration one half hour before and one hour after treatment. The resident physician observed the child before and after treatment and recorded the effects.

* From the Babies' Hospital.

1. Blackfan and Macy: The Intraperitoneal Injection of Saline Solution, Am. J. Dis. Child. 15:19, 1918. 
Only infants showing signs of dehydration were given treatment. Seventy-six infants received 269 injections of fluid. Of these, 155 were hypodermoclyses; ninety-two were intraperitoneal injections, and twenty-two were sinus injections. The mortality in these seventy-six cases was 56.5 per cent.

The amount of the clyses varied between 90 and 150 c.c., depending on the size and condition of the child. In a few cases $1 / 1,000$ to $1 / 500$ grain of atropin was used in the clysis solution. On one occasion, 120 c.c. of a 6 per cent. dextrose solution was used. The intraperitoneal injection fluids were of various formulas, and no attempt is made here to demonstrate different results from different solutions. The amounts varied between 150 and 240 c.c.

The following solutions were used: 6 per cent. dextrose in physiologic sodium chlorid solution; 6 per cent. dextrose in distilled water; physiologic sodium chlorid solution; 2 per cent. sodium bicarbonate with 2 per cent. dextrose solution; 1 per cent. sodium bicarbonate with 1 per cent. dextrose solution.

Fig 1.-Ty.pe of chart headings used in all cases.

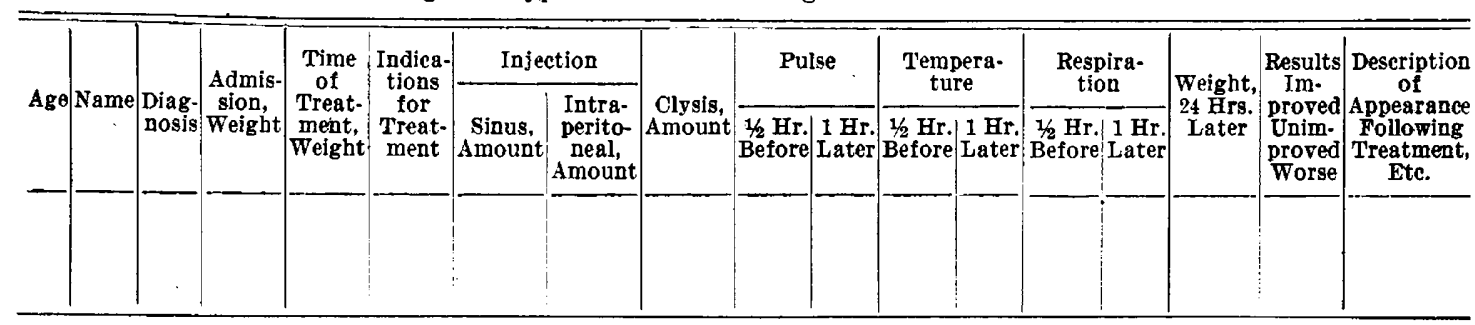

We have not had any bad results from the intraperitoneal injections, although we have noted discomfort in a few cases.

The clinical diagnoses made in these cases were as follows: Acute ileocolitis, ten cases; acute intestinal intoxication, twenty-six cases; marasmus, eight cases; bronchopneumonia, two cases; feeding cases, fourteen cases; gastro-enteritis, six cases; rumination, pyelitis, marasmus and congenital syphilis, marasmus with furunculosis and peritonitis, marasmus with otitis media, enteritis with bronchopneumonia, hydrocephalus, ileocolitis with bronchopneumonia, acute enteritis and gastro-enteritis with acidosis, one case of each.

In an analysis of the seventy-six cases, there were twenty-six cases of acute intestinal intoxication, or 34.2 per cent. ; of all other diseases there were fifty cases, or 65.8 per cent. The mortality in the intestinal intoxication cases was 76.9 per cent., as contrasted with 52 per cent. mortality in other diseases.

In the entire group of cases, 56.5 per cent. of the patients died; 5.2 per cent. were discharged improved; 27.6 per cent. were cured; 
5.2 per cent. were removed from hospital against advice; 3.9 per cent. died within a period of two or three days following their discharge from hospital; 1.3 per cent. were discharged unimproved.

The Relation Between the Number of Injections Given Each Child and the Mortality Curve.-In going over these records one is impressed with the bad results in those infants who received three or more injections as compared with those who received less than three injections. In spite of the results noted in Figure 2, it has been found that repeated injections do no harm. One of our patients received as many as thirteen.

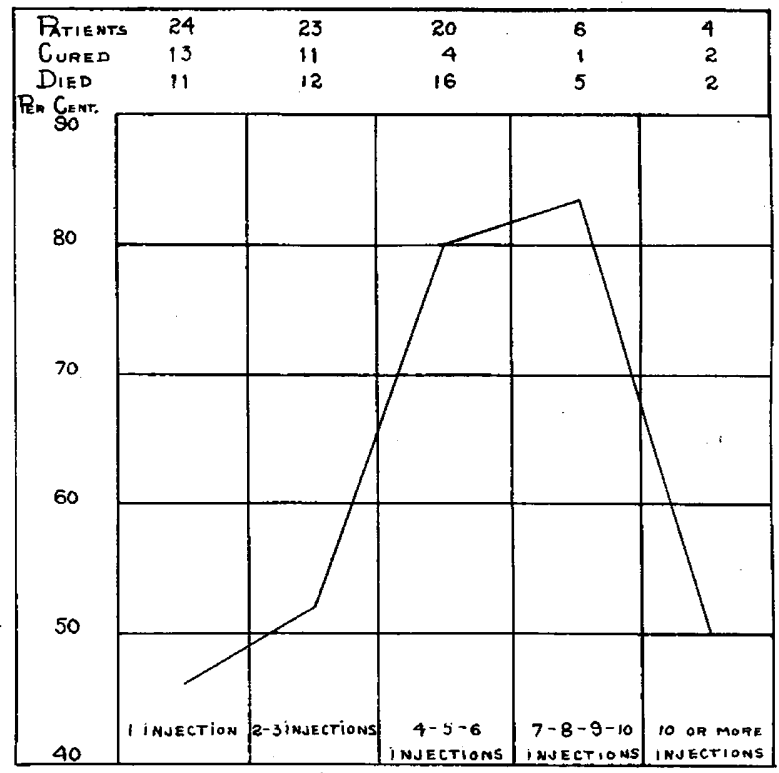

Fig. 2,-Relation between number of injections and mortality.

This series of cases has been grouped into five divisions. In Group 1 are those infants who received one injection. In this group were twenty-four infants, eleven of them died, a mortality of 45.8 per cent. In Group 2 are those infants who received two or three injections, twenty-three cases, with a mortality of 52.1 per cent. Group 3 includes those who had four, five or six injections, twenty in all, with a mortality of 80 per cent. Group 4 includes those infants who received seven, eight, nine or ten injections, six cases, with a mortality of 83.3 per cent. In Group 5 are thlose who had ten or more injections, four cases, with a mortality of 50 per cent. One notes the lower death rate in the group which had three injections or less as compared with the higher rate in the group which had from four to ten injections.

We are not prepared to say what factors, other than water reten- 
tion, influenced the results in Group 1. There are so many conditions which might influence these figures that any conclusions drawn would be purely speculative.

The Relation Between the Weight of the Child and the Mortality Rate.-A glance over these weight records will convince one that the class of cases was not a favorable one. It has been assumed in the past that a very small infant did not react so well to fluid injection as a larger infant. A study has been made here to discover whether this assumption was correct. The infants have been divided into 5 groups.

Group 1, from 2,000 to $3,000 \mathrm{gm}$. weight.

Group 2, from 3,000 to $4,000 \mathrm{gm}$. weight.

Group 3, from 4,000 to $5,000 \mathrm{gm}$. weight.

Group 4, from 5,000 to $6,000 \mathrm{gm}$. weight.

Group 5, 6,000 and above.

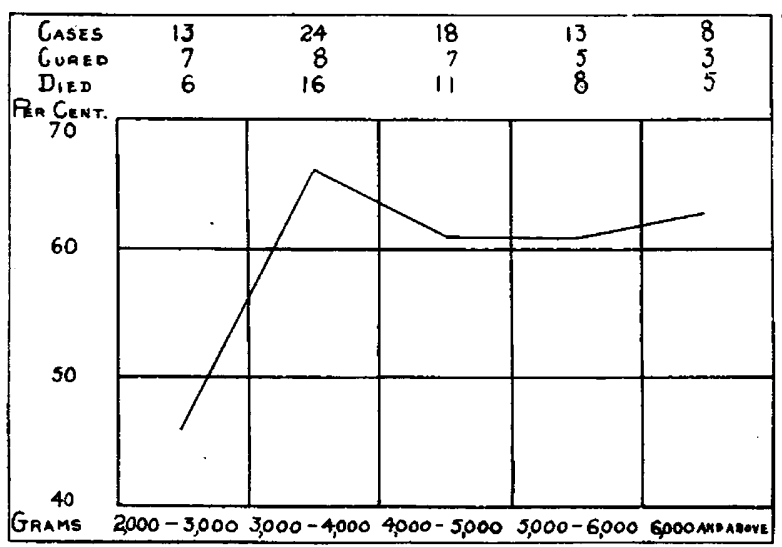

Fig. 3.-Relation of weight to mortality.

It may be noted that the mortality was lowest among the smallest infants, but highest in the next smallest or those weighing from 3,000 to $4,000 \mathrm{gm}$. If the infants were divided into two groups, and those weighing less than $5,000 \mathrm{gm}$. placed in one group, and those weighing more than $5,000 \mathrm{gm}$. were placed in another group, it is found that the mortality in the first group is 57 per cent., and in the second group 61 per cent. From these figures one concludes that there is no definite relation between the weight of the infant and the mortality rate.

It may be noted throughout our series of cases that the weight of the infant bore no exact relation to the amounts of fluid given at each injection. The smallest infants had the lowest mortality rate. It may be possible that they received more nearly their fluid requirements.

The Mortality Curve in Sixty-One Cases with Reference to the Infant's Ability to Retain the Fluid of the First Fere Injections.-Our 
only means of measuring this was by noting the change in weight following injections. As the infants also received food and fluids by mouth during the same period in which fluid injections were given it will be noted that there were other factors which might have influenced these figures.

The cases have been divided into two groups. In the first group are placed those infants who maintained their body weight or gained weight during the twenty-four hours following the injection of fluids. These include cases in which fluids were given either by clyses, intraperitoneally or by the intrasinus route. The mortality in the first group was 46.6 per cent.

In the second group are those infants who showed a weight loss twenty-four hours after injection. In this group the mortality was 70 per cent.

TABLE 1.-Relation of Weight Twenty-Four Hours Following Injection of Fluid to Mortality

\begin{tabular}{|c|c|c|c|c|c|c|}
\hline I. & 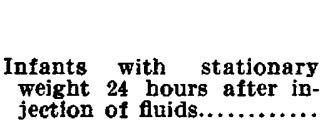 & Cases & Recovered & Died & Mortality & $\begin{array}{l}\text { Mortality I and II } \\
\text { (combined) }\end{array}$ \\
\hline II. & 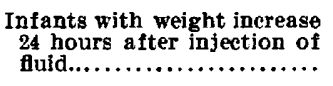 & 23 & 11 & 12 & $52.1 \%$ & $40 \%$ \\
\hline III. & 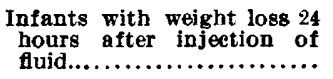 & 31 & 8 & 22 & $71 \%$ & \\
\hline
\end{tabular}

The Relation Between Age and Mortality.-The seventy-seven cases have been divided into four groups:

Group 1, up to and including 3 months of age.

Group 2, from 3 to 6 months of age.

Group 3, from 6 to 12 months of age.

Group 4, 12 months and older.

There was no marked difference in the death rate of the different groups, but it was slightly lower in the youngest group, namely, 52 per cent.

The Effect on Temperature, Pulse, Respiration and Weight of the Different Kinds of Fluid Injections.-Pulse, respiration and temperature records were taken one half hour before and one hour after injection. The infant was weighed before injection and twenty-four hours afterward. As the vital signs are so easily altered in infants, we have considered a change of ten in pulse rate, five in respiration and of one degree $F$. in temperature as being definite signs of a reaction. Variations less than these we have not recorded. We have considered $25 \mathrm{gm}$. as being a definite weight change, any change less than $25 \mathrm{gm}$. we have not recorded. 
Intrasinus Injections. - Twenty-one infants received intrasinus injections; nineteen of these received 90 c.c. of a 6 per cent. dextrose solution and two received 90 c.c. of a 4 per cent. sodium bicarbonate solution. In ten of these cases, or 47.6 per cent., there was a rise in pulse rate; in seven, or 33.3 per cent., the pulse showed no change, and in four cases, or approximately 19.1 per cent., there was a fall in the pulse rate.

Eight infants, or 38 per cent., had a rise in respiratory rate; in twelve, or 57 per cent., the rate was unchanged, and in one case there was a fall in the respiratory rate.

Eight infants, or 38 per cent., had a rise in temperature; eight, or 38 per cent., had a fall in temperature, and four showed no change.

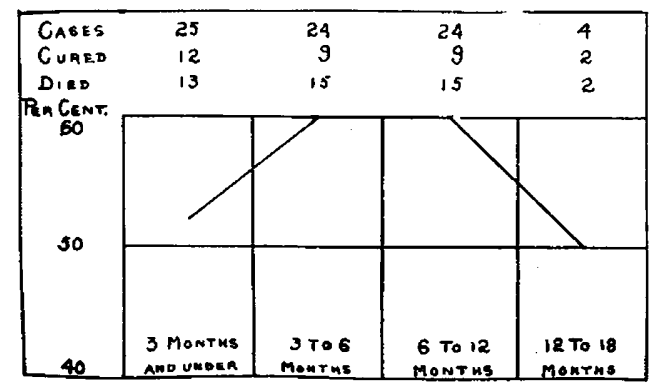

Fig. 4.-Relation of age to mortality.

Of twenty infants, four, or 20 per cent., had a gain in weight; eleven, or 55 per cent., lost in weight, and the remainder showed no change.

Intraperitoneal Injections.-Ninety-nine infants received intraperitoneal injections; forty-seven, or 52.7 per cent., had a rise in pulse rate; nine, or 10.1 per cent., had a fall in pulse rate, and in thirty-four, or 37.7 per cent., no change was noted. In eighty-seven intraperitoneal injections, forty, or 45.9 per cent., had a higher respiratory rate following injection; two, or 2.3 per cent., had a lower rate, and in forty-five, or 51.7 per cent., there was no change. In eighty-six cases studied with reference to effect on the temperature, it was noted that in twentyseven infants, or 31.6 per cent., there was a rise in temperature following injection; in nineteen, or 21 per cent., there was a fall in temperature, and in forty, or 47.4 per cent., no change was noted. In sixty-five cases studied with reference to the effect on body weight, it was noted that twenty-three infants, or 35 per cent., showed a weight gain twenty-four hours following injection; twenty-six, or 40 per cent., lost weight, and in sixteen, or 25 per cent., there was no change in weight. 
Hypodermoclyses. - One hundred and forty-five infants received hypodermoclyses; thirty, or 20.7 per cent., had a more rapid pulse rate; nine, or 6.2 per cent., had a fall in pulse rate, and in 106, or 73.1 per cent., there was no change in the rate.

Of 140 injections, 28 infants, or 20 per cent., had a rise in the respiratory rate; eight, or 5.7 per cent., had a fall in rate, and in 104, or 74.3 per cent., there was no change of rate. Of 140 injections, thirty-eight infants, or 26.9 per cent., had a rise in temperature; nineteen, or 13.9 per cent., had a fall in temperature, and in eighty-three, or 58.9 per cent., the temperature was not affected. Of 131 infants, forty-two, or 32 per cent., showed an increase in weight; in sixty-four, or 48.8 per cent., there was a weight loss, and in twenty-five, or 19 per cent., there was no change in weight. The records in a few cases were incomplete.

A comparison of the effect on pulse, respiration, weight and temperature of the three methods of introducing fluids shows the following:

Pulse: In 47.6 per cent. there was a rise in pulse rate after the sinus injection; in 52.2 per cent. after the peritoneal injection, as compared with a rise of only 20 per cent. after clyses. In 73.1 per cent. of the tabulated cases there was no rise in the pulse rate following the injection of fluid under the skin.

TABLE 2.-Effect on Pulse, Respiration, Temperature and Weight

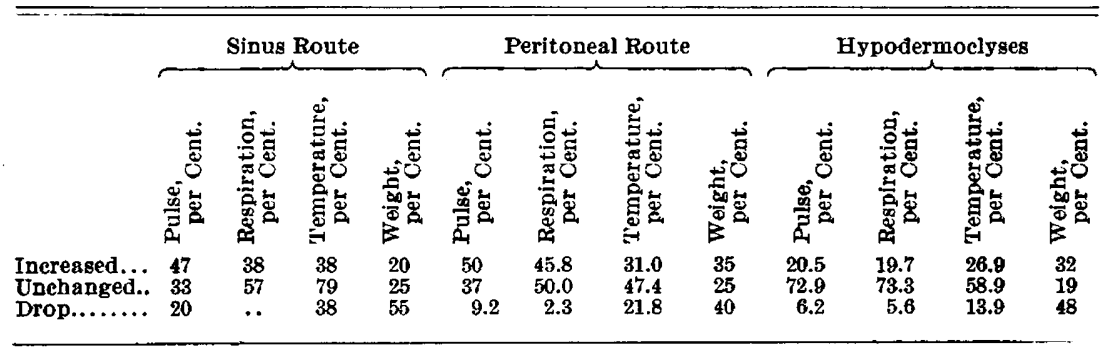

Respiration: In 38 per cent. respiration was increased following sinus injection as compared with 45.9 per cent. following intraperitoneal injection, and only 20 per cent. after hypodermoclyses. In the intraperitoneal injection a mechanical factor may be operative which has an influence on respiration.

Temperature: In 38 per cent. rise in temperature followed sinus injection as compared with 31 per cent. following intraperitoneal injection, and 26.9 per cent. following hypodermoclyses.

Weight: In 20 per cent. a gain in weight was noted after sinus injection; in 35 per cent. after intraperitoneal injection, and in 32 per cent. after hypodermoclyses. 
Figure 5 shows the daily temperature and humidity for July, August and part of September, 1918, indicating the dates of the fortythree deaths which occurred among the patients of this study. We have done this to learn whether there was any relationship between the mortality and weather conditions. The patients are too few in number to serve as a basis of conclusions of this nature. There was a group of six deaths on August 16,17 and 18. There deaths occurred during a falling temperature and humidity, the humidity on August 16 being 39 , the lowest point reached that summer. The temperature on

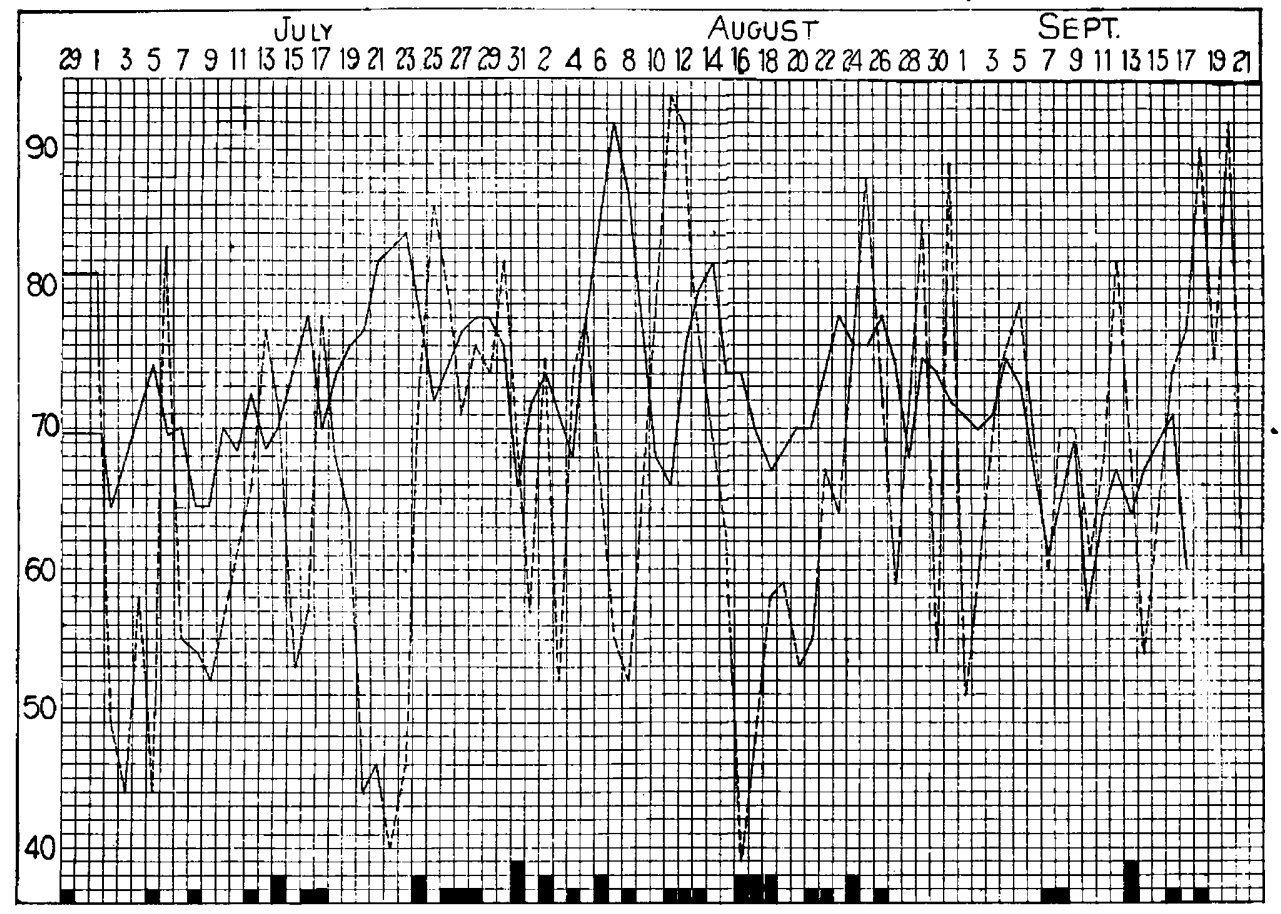

Fig. 5.-Relation of mortality to temperature and humidity. Each black square represents one death. Broken line represents relative humidity; solid line represents temperature.

August 16, 17 and 18 was 70 and $66 \mathrm{~F}$. on the three successive days. This single observation is in keeping with our experience that the sudden fall in temperature and humidity occurring during a period of hot weather has a more harmful effect on the feebler infants than the waves of high temperature and humidity.

Mortality and Duration of Symptoms.-In only thirty-three of sixty-eight cases was treatment begun earlier than ten days after the onset of symptoms. In these thirty-three cases the mortality was 54 per cent. In thirty-five cases the treatment was begun ten or more 


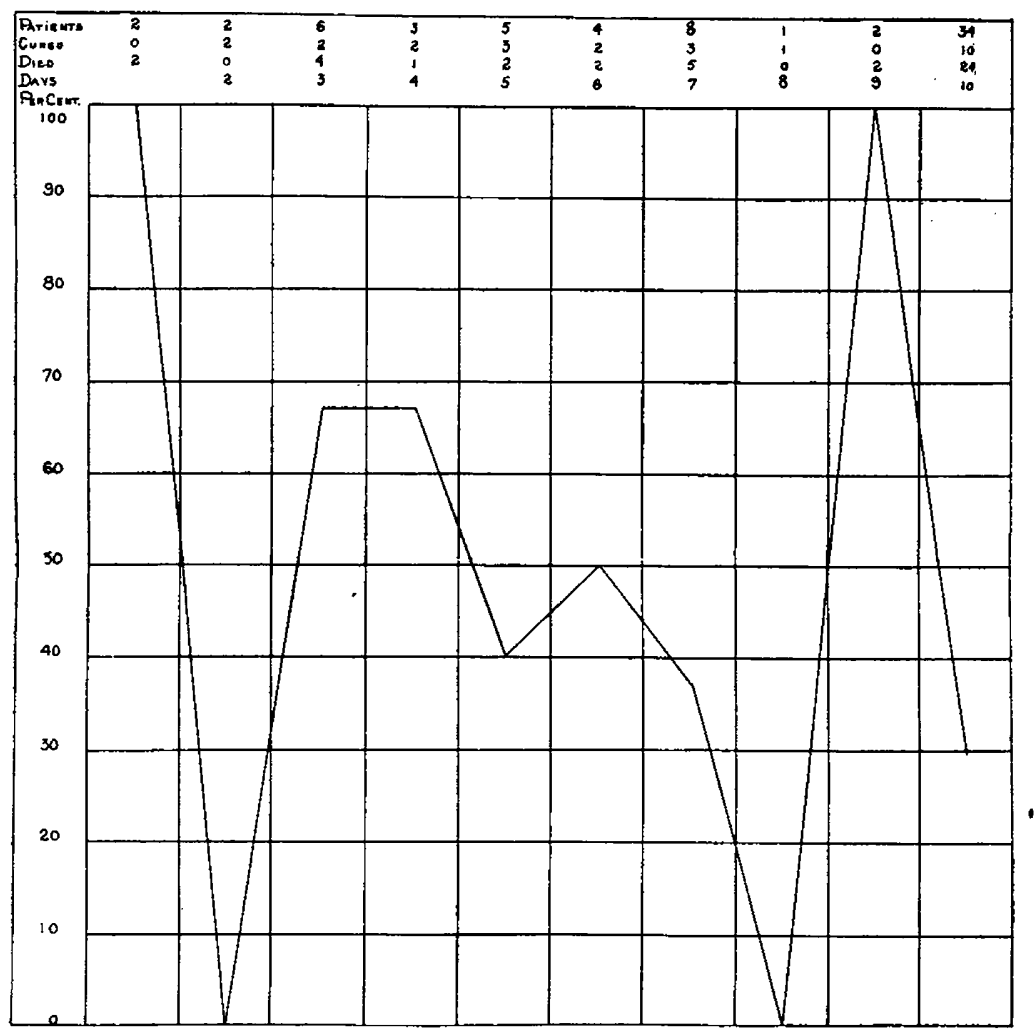

Fig. 6.-Relation of mortality to duration of symptoms.

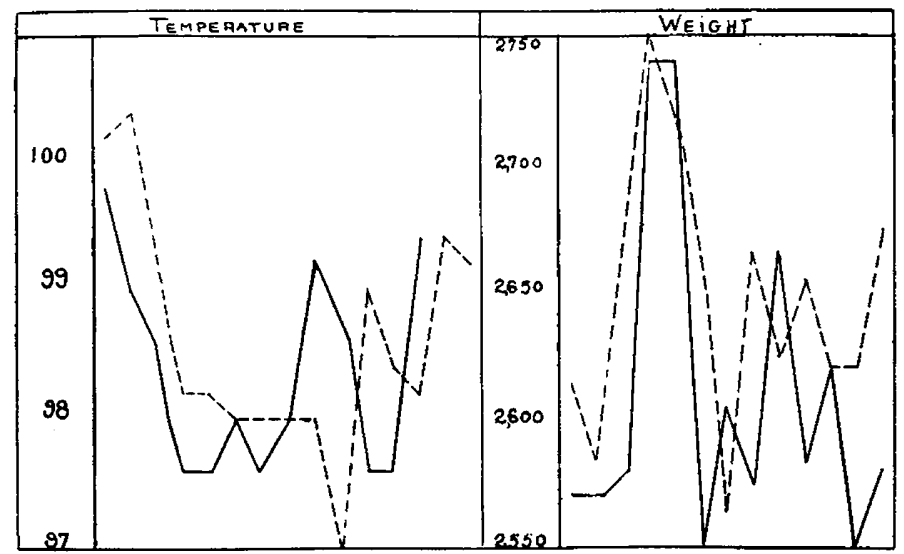

Fig. 7.-Clinical record of a patient who received thirteen saline hypodermoclyses of 90 c.c. each. The solid line represents the temperature one-half hour before injection of fluid and weight at time of injection of fluid. The broken line represents the temperature one-half hour after the injection of fluid and the weight twenty-four hours after injection. 
days after onset of symptoms, and in this group the mortality was 68 per cent. It is to be regretted that more cases were not received for admission directly after the onset of symptoms. One of us has recently had a patient admitted to the hospital on the third day after the onset of symptoms. He showed definite evidence of great fluid loss. This child, 20 months old, had his attack begin with diarrhea

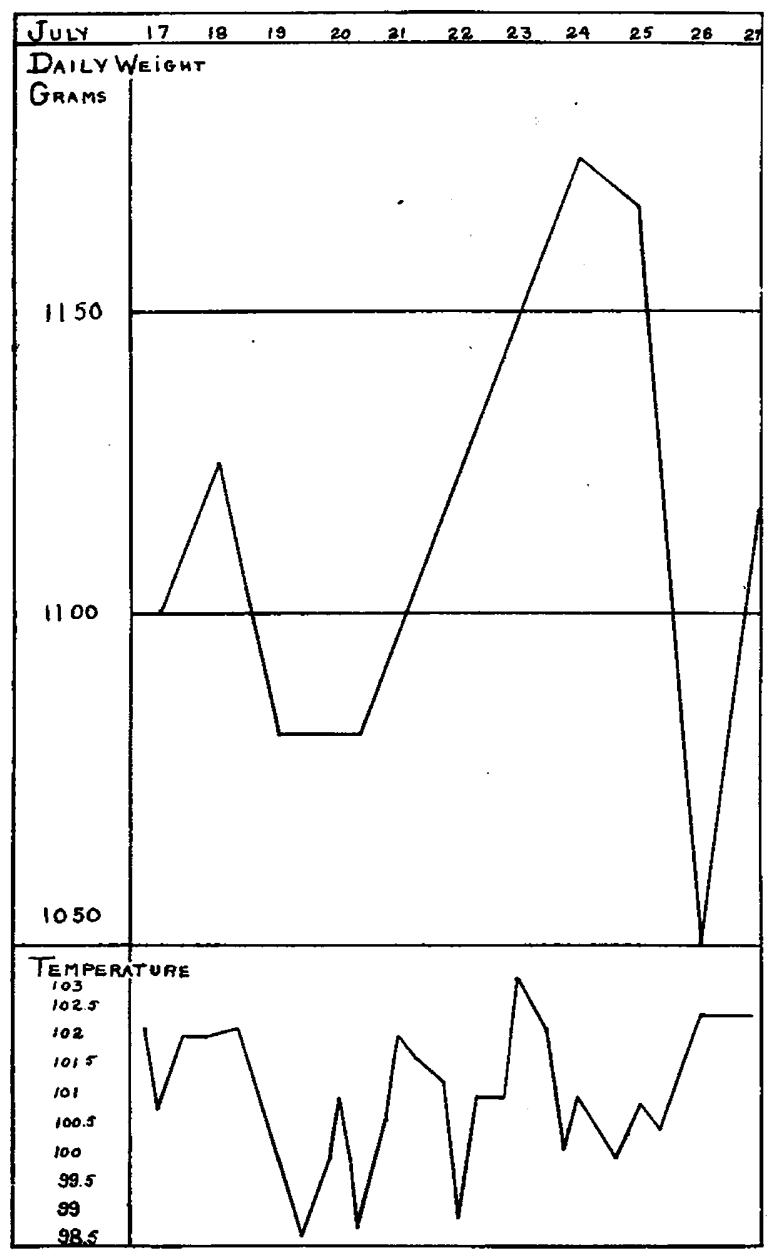

Fig. 8.-Effect produced on weight and temperature by daily intraperitoneal injections of 60 c.c. of a 4 per cent. sodium bicarbonate solution.

and vomiting. His weight prior to the onset was 27 pounds. On the third day, he suddenly became extremely prostrated, and was apparently moribund when admitted to the hospital. The patient was in extreme shock. During a period of eight hours, about 1,600 c.c. of fluid was administered, as follows : 1,200 c.c. physiologic sodium chlorid 
solution by clysis; 100 c.c. 5 per cent. sodium solution intravenously; 90 c.c. 5 per cent. dextrose solution intravenously, and 250 c.c. physiologic sodium chlorid solution intraperitoneally. Following directly on this treatment, his condition improved and he was discharged from the hospital apparently well eight days later.

Figure 7 shows graphically the course of one case in which we gave thirteen hypodermoclyses. The patient was cured.

Figure 8 represents the intraperitoneal injections given a rabbit weighing $1,100 \mathrm{gm}$. It was a male animal apparently normal. The diet consisted of lettuce, oats, carrots and bread, and a constant supply of water was allowed. The rabbit was given intraperitoneally 60 c.c. of a 4 per cent. sodium bicarbonate solution daily for eleven successive days. The temperature was taken three times a day, and daily weighings were made. The animal was as active as the controls during the periods of observation, and the appetite was not affected. His weight on the eleventh day was $24 \mathrm{gm}$. more than on the day the injections were begun. The weather was intensely hot, and the animal was caged in a small room. Some of the controls, as well as the experimental animal had fever during this same period.

\section{SUMMARY}

Injection of fluid into the peritoneal cavity in dehydrated infants is a simple method of procedure and in our hands has had no undesirable effects.

The size of the infant is no bar to his ability to utilize injected fluids.

From the results of injections in very small infants, weighing less than $3,000 \mathrm{gm}$., we are led to believe that larger infants might utilize larger amounts than have been given to infants in this study.

Age has no bearing on the infant's ability to utilize injected fluids.

The pulse is more frequently affected after sinus and intraperitoneal injections than after hypodermoclyses.

The respiratory rate is more frequently affected in peritoneal injections than in sinus injections and hypodermoclyses.

The temperature is more frequently elevated in sinus injections than in intraperitoneal injections or hypodermoclyses.

Weight gains are more frequently noted following intraperitoneal injections than after sinus injections or clyses.

Certain infants do not show any improvement until they have had repeated injections of fluid.

The shorter the interval between the onset of symptoms and the beginning of treatment the greater is the response. 